\title{
Références bibliographiques du dossier « L'école et son contrôle »
}

Cécile de Bouttemont

\section{OpenEdition \\ Journals}

Édition électronique

URL : http://journals.openedition.org/ries/459

DOI : $10.4000 /$ ries.459

ISSN : 2261-4265

Éditeur

Centre international d'études pédagogiques

\section{Édition imprimée}

Date de publication : 1 septembre 2008

Pagination : 137-147

ISBN : 978-2-85420-573-2

ISSN : $1254-4590$

Référence électronique

Cécile de Bouttemont, «Références bibliographiques du dossier "L'école et son contrôle » », Revue internationale d'éducation de Sèvres [En ligne], 48 | septembre 2008, mis en ligne le 28 juin 2011, consulté le 26 avril 2021. URL : http://journals.openedition.org/ries/459 ; DOI : https://doi.org/ $10.4000 /$ ries.459 


\title{
Références bibliographiques
}

\section{Cécile de Bouttemont}

Pour établir la bibliographie de ce dossier, il est apparu d'emblée qu'il serait difficile de dissocier complètement les notions de contrôle et d'évaluation. Certes, elles sont distinctes mais elles obéissent à la même finalité et sont toutes deux considérées comme des outils au service d'une politique. De fait, la grande majorité de la production éditoriale aborde les deux notions sans établir nécessairement une claire séparation. Par ailleurs, la notion de contrôle est prise dans un faisceau de concepts tels que le "pilotage», la "régulation» ou la "gouvernance».

Cette bibliographie, non exhaustive, recensera donc des rapports, des ouvrages et des articles sur cet ensemble de notions. Les références, pour l'essentiel publiées depuis moins de dix ans, sont regroupées en deux parties. La première s'attache à décrire les modalités d'exercice du contrôle et de l'évaluation des acteurs du système éducatif. Les références rassemblées portent sur le contrôle et l'évaluation des acquis des élèves, que ce soit au niveau national ou international, de l'évaluation des enseignants et plus largement des établissements d'enseignement. La seconde partie rassemble des références sur le contrôle et l'évaluation en tant qu'outils de fonctionnement de l'école. Ces ouvrages tentent de définir les liens entre contrôle et pilotage et approfondissent la problématique de la gouvernance des politiques éducatives.

Bibliographie arrêtée le 15 juin 2008

\section{Contrôle et ÉVALUation des ACTEURS DU SYSTÈME ÉDUCATIF}

\begin{abstract}
BEHRENS Matthis dir., La qualité en éducation: pour réfléchir à la formation de demain, Presses de l'Université de Québec/Sainte Foy, 2007

Le concept de qualité est de plus en plus utilisé pour évaluer le rapport de conformité entre mandat, normes et standards d'une part et la réalité de l'interaction formatrice d'autre part. La qualité devient aussi un moyen de juger la performance d'un système, de le piloter et de contrôler si l'école remplit son contrat. Quelles données faut-il rassembler pour rendre compte de son efficacité, de son efficience et de son équité? Est-il possible de mettre en œuvre les innovations et les réformes sans l'appui des enseignants et des formateurs?
\end{abstract}

CHOISNARD Marie-Françoise, GAUTHIER Roger-François, KERRERO Christophe et al. "Les évaluations», Les Cahiers de l'Éducation, octobre 2006, $n^{\circ} 58$, pp. 4-14 Les cinq articles de ce dossier invitent à réfléchir aux enjeux et aux limites de l'évaluation appliquée aux différents niveaux du système éducatif ainsi qu'à l'utilisation qui en est faite.

FIGARI Gérard, Évaluer: quel référentiel?, De Boeck Université/Bruxelles, 1994, 191 p., (pédagogies en développement)

Enseignant en sciences de l'éducation, l'auteur présente ici une méthodologie d'évaluation adaptée à la diversité des situations et des enjeux. Après avoir analysé la notion de référentiel, il montre, en considérant surtout le projet éducatif, comment retrouver un référentiel par l'analyse des situations éducatives. Il explique ensuite en quoi l'évaluation diagnostique se distingue de l'audit, avant d'exposer la façon dont s'élabore la construction d'un référentiel pour divers types d'évaluation comme celle de l'établissement scolaire ou d'un dispositif de formation. 
FIGARI Gérard, GRANGEAT Michel, GAUTIER Gildas et al., "L'évaluation du curriculum: numéro thématique », Mesure et évaluation en éducation, septembre 2003, vol. 26, $n^{\circ} 3,83$ p., bibliogr.

Le curriculum est au centre des préoccupations de toutes les réformes éducatives. Il est devenu l'un des objets d'étude les plus importants en sciences de l'éducation. De nombreuses questions sur les contenus, les modalités et les dispositifs d'enseignement sont abordées dans les quatre articles de ce numéro.

FIGARI Gérard, MOTTIER LOPEZ Lucie, Recherche sur l'évaluation en éducation: problématiques, méthodologies et épistémologie, L'Harmattan/Paris, 2006, 257 p., bibliogr. (Évaluer)

Les contextes, les pratiques et les connaissances concernant les processus d'évaluation évoluent depuis une vingtaine d'années. Vingt-six auteurs belges, canadiens, français, luxembourgeois, portugais et suisses présentent chacun un état de la recherche dans un domaine de l'activité d'évaluation. L'ouvrage s'organise autour de trois axes: la diversification des problématiques de l'évaluation; l'approfondissement des méthodologies de l'évaluation; l'évolution du statut épistémologique de l'évaluation.

\section{LES ÉLÈVES}

BARDI Anne-Marie, BOUZAHER Myriem, DYCKMANS Annie et al., Les acquis des élèves, pierre de touche de la valeur de l'école?, ministère de l'Éducation nationale, de l'enseignement supérieur et de la recherche, juillet 2005, rapport $n^{\circ}$ 2005-079, 85 pages téléchargées du site http://lesrapports.ladocumentation francaise.fr

L'évaluation des acquis des élèves et le pilotage par les résultats permet-il d'améliorer l'efficacité du système éducatif? Dans ce rapport, l'évaluation des acquis des élèves est envisagée comme un référentiel à partir duquel interroger l'ensemble du système éducatif.

\section{BOTTANI Norberto, La France et les évaluations internationales, Haut Conseil de l'évaluation de l'école/Paris, 2005, 166 p.}

Les enquêtes internationales sur l'évaluation des acquis des élèves permettent de comparer les résultats, de s'inspirer d'expériences éducatives, des cursus et des programmes d'enseignement d'autres pays. Le rapport s'intéresse notamment à l'historique de ces enquêtes, à leur élaboration, leur mise en œuvre, à leurs coûts et à leurs financements.

BROADFOOT Patricia ed., SHEN Ce, DAVIES Peter et al., "Dynamic versus arbitrary standards: recognising the human factor in assessment», Assessment in education, juillet 2002, vol. 9, pp. 157-268, bibliogr.

Les articles réunis dans ce numéro se proposent de montrer l'importance d'appréhender les données en évaluation dans leur contexte culturel. Malgré l'effort scientifique d'objectivité qui sous-tend les techniques d'évaluation, ces dernières sont toujours le résultat de l'interaction de personnes spécifiques et de contextes temporel et spatial précis. Les auteurs mettent en évidence une tension entre des "critères arbitraires» et des "critères dynamiques» à travers des exemples empruntés notamment à la géographie, aux mathématiques, à l'évaluation des élèves et à celle des réformes éducatives, à la fois dans des comparaisons internationales et à l'échelle des pays. 
CEARD Marie-Thérèse, REMOND Martine, VARIER Michelle, L'appréciation des compétences des élèves et des jeunes en lecture et en écriture et l'évolution de ces compétences dans le temps: rapport établi à la demande du Haut Conseil de l'école, décembre 2003, 147 pages téléchargées du site: http://cisad.adc. education.fr/hcee (Évaluations françaises et internationales)

Le rapport étudie l'ensemble des dispositifs d'évaluation en France ainsi que les dispositifs étrangers ou internationaux, en matière de compétence en lecture et en écriture dans l'enseignement primaire et au collège. Il examine, entre autres, l'évolution des compétences à partir de 1979, date des premières évaluations mises en place par le ministère de l'Éducation nationale.

HOUCHOT Alain, ROBINE Florence, Les livrets de compétences: nouveaux outils pour l'évaluation des acquis - Rapport à monsieur le ministre de l'Éducation nationale, Inspection générale de l'Éducation nationale/Paris, 2007, $60 \mathrm{p}$.

La Loi d'orientation et de programme pour l'avenir de l'École d'avril 2005 a institué en France le «socle commun de connaissances et de compétences». Pour l'accompagner, un livret personnel de l'élève a été mis en place. Il permet de suivre l'acquisition progressive des compétences. Le rapport s'intéresse aux conditions dans lesquelles ce livret peut devenir un outil efficace de l'évaluation des acquis des élèves. Après avoir rappelé la finalité de cette forme d'évaluation, les auteurs présentent des exemples d'évaluation des compétences dans plusieurs pays européens - Belgique, Angleterre, Portugal, Suisse, France - et du Québec. Au-delà de la modification des pratiques d'évaluation, les auteurs préconisent un profond changement dans les représentations et les pratiques pédagogiques.

IGEN: Inspection générale de l'Éducation nationale, IGAENR: Inspection générale de l'administration de l'Éducation nationale, Évaluation des enseignements et des pratiques enseignantes, évaluation des acquis des élèves IN Rapport annuel des inspections générales, Paris: Documentation française, 2005, p. 61-101

À quoi sert l'évaluation des acquis des élèves? Qui s'en sert? Pour quoi et comment? L'un des chapitres porte sur cette "question nouvelle pour de nouveaux enjeux». L'évaluation doit devenir consubstantielle au pilotage du système éducatif.

\section{LESSARD Claude, MEIRIEU Philippe, L'obligation de résultats en éducation:} évolutions perspectives et enjeux internationaux, De Boeck/Bruxelles, 2005, 327 .

Cette publication reprend les débats tenus lors des Entretiens Jacques-Cartier à l'université de Montréal en 2000. Les dix-neuf auteurs, issus d'univers professionnels très différents, esquissent une amorce de réponse aux inquiétudes et aux questions soulevées par l'obligation de résultats. Celle-ci y est définie de plusieurs manières, en référence aux apprentissages des élèves, en tant que responsabilité collective, en rapport avec les moyens, processus ou procédures de travail et en fonction des compétences de l'enseignant. Les contributeurs prennent également en compte les tensions entre l'accent mis sur les cibles quantitatives et une approche qualitative de la réussite scolaire et éducative.

\section{MINISTĖRE DE L'ÉDUCATION NATIONALE, L'état de l'école n' 17: 30 indi- cateurs sur le système éducatif français, DEPP/Paris, 2007, 77 p.}

Parmi les indicateurs proposés dans cette dix-septième édition, ceux qui concernent la performance des élèves s'appuient sur les évaluations nationales et internationales des acquis des élèves. 
OCDE: Organisation de coopération et de développement économiques/Paris, PISA 2006: les compétences en sciences, un atout pour réussir. Volume 1: analyse des résultats, OCDE/Paris, 2007, 419 p., bibliogr.

La dernière enquête de l'OCDE, programme international pour le suivi des acquis des élèves (PISA), a pour objet majeur l'évaluation de la culture scientifique mais elle s'est également penchée sur les mathématiques et sur la compréhension de l'écrit. Le rapport présente les résultats du cycle PISA 2006 et retrace l'évolution depuis 2000 des pays qui ont participé à cette enquête.

OCDE: Organisation de coopération et de développement économiques/Paris, PISA 2006. Volume 2: données, OCDE/Paris, 2007, 310 p.

L'ensemble des données du cycle PISA 2006 est exposé dans ce volume sous forme de tableaux synthétiques. Ils fournissent ainsi un état des lieux détaillé de l'apprentissage des sciences aujourd'hui, examinant non seulement les performances des élèves, mais aussi leurs attitudes et la relation entre leurs performances.

\section{PERRENOUD Philippe, L'évaluation des élèves: de la fabrication de l'excellence à la régulation des apprentissages. Entre deux logiques, de Boeck Universitél} Bruxelles/Belgique, 1998, 219 p., bibliogr., (Pédagogies en développement)

L'auteur s'attache à décrire l'évaluation des élèves. Selon lui, la notion est partagée entre une logique certificative au service de la sélection et, depuis 1970, une logique formative au service des apprentissages. Il propose une évaluation orientée vers la régulation mais cela ne peut se faire sans remise en question du contrat pédagogique, de la gestion de classe, de la nature du curriculum et de l'excellence scolaire. À travers des textes déjà publiés ou inédits, l'ouvrage évoque l'oscillation de l'évaluation entre ces deux logiques coexistantes et insiste sur le fait que le véritable enjeu de l'évaluation est la lutte contre l'échec scolaire.

POSTLETHWAITE Neville, Le pilotage des résultats des élèves, IIPE/Paris, 2005, 163 p., bibliogr.

Les pays sont de plus en plus nombreux à participer à des études sur le pilotage des résultats des élèves, démarche qui leur permet d'orienter leur politique éducative et de promouvoir les réformes susceptibles d'améliorer les performances de leur système éducatif. À l'aide d'exemples tirés d'études internationales, l'ouvrage explique notamment l'importance du pilotage, son évaluation et ses conséquences pour les responsables de l'éducation.

BOUVIER Alain coord., "Que savent les élèves?", Revue internationale d'éducation Sèvres, décembre 2006, n 43, 179 p.

Privilégie-t-on les connaissances ou les compétences? Comment les priorités nationales sontelles mises en œuvre et évaluées? Quel rôle cette évaluation joue-t-elle dans la formation de l'élève? Quelles sont les informations utiles aux enseignants pour préparer leur action quotidienne? Et surtout, quelle est la place de l'élève dans ces différents dispositifs? Aborder ces questions, c'est s'intéresser aux résultats de l'école. Ce dossier présente les stratégies déployées et les réponses apportées dans neuf pays: États-Unis, Singapour, Japon, Brésil, France, Allemagne, Angleterre, Finlande, Portugal, ainsi qu'une réflexion sur la conception et l'usage des outils actuels d'évaluation.

\section{ROEGIERS Xavier, L'école et l'évaluation: des situations pour évaluer les compé-} tences des élèves, de Boeck Université/Bruxelles, 2004, 367 p., bibliogr., index

L'auteur pose tout d'abord la problématique de l'évaluation au sein des systèmes éducatifs: son lien aux valeurs, ses enjeux, et les risques qu'elle comporte. Ensuite, à partir de nombreux exemples, il propose des outils le praticien pour l'aider à construire une épreuve d'évaluation, corriger les copies. 
SUCHAUT Bruno, Les évaluations nationales en France: outils pédagogiques et outils de pilotage, intérêts, limites et nouvelles perspectives, $19^{e}$ colloque de l'ADMEE EUROPE (11-13 septembre 2006, Luxembourg), Université de Bourgogne et IREDU C.N.R.S., Dijon, 17 pages téléchargées du site http://halshs. ccsd.cnrs.fr

L'auteur expose une analyse des épreuves des évaluations nationales et cherche à montrer en quoi les outils d'évaluation livrent des informations capitales sur les apprentissages réalisés par les élèves à condition de ne pas se limiter à la seule approche institutionnelle, notamment à la classification des compétences élaborée par le concepteur.

\section{LES ENSEIGNANTS}

«L'inspection en questions », Administration et Éducation, 2005, $n^{\circ} 108-4,211$ p.

Ce numéro cherche à rendre compte des multiples interrogations dont les corps d'inspection sont aujourd'hui l'objet. Créés au départ pour inspecter des individus dans une perspective de gestion de carrière, les inspecteurs sont désormais de plus en plus sollicités pour inspecter des disciplines ou des établissements. Les questions de la place de l'élève, de l'efficacité de l'enseignement qui lui est dispensé sont ainsi abordées par les différents contributeurs.

\section{LADERRIERE Pierre, BRESSOUX Pascal, PAQUAY Léopold, "Quelle évaluation} des enseignants au service de l'école?", Actes du séminaire 2007, 6-7 décembre 2007, Leysin/Suisse, 118 p., http://publications.irdp.relation.ch/ftp/1213272596081.pdf

Le séminaire, qui porte sur l'évaluation des enseignants et s'interroge sur la tâche de l'inspecteur et du directeur d'établissement, cherche à établir la finalité de cette évaluation qui n'est pas faite pour elle-même mais au service de l'école. Huit ateliers s'inspirant des expériences réalisées en Suisse illustrent les facettes de cette problématique.

\section{PAQUAY Léopold dir., L'évaluation des enseignants: tensions et enjeux, L'Harmattan/Paris, 2004, 334 p.}

L'objectif de cet ouvrage est de faire le point sur les pratiques d'évaluation des enseignants dans divers pays et de fournir des outils d'analyse qui permettent d'en dégager les enjeux. Ainsi les différents contributeurs tentent-ils d'évaluer les conditions dans lesquelles les évaluations des enseignants peuvent améliorer la qualité de l'enseignement et permettre une bonne régulation du système éducatif. Une première partie définit la problématique générale et fournit quelques outils d'analyse, la seconde partie explore les pratiques diversifiées d'évaluation des enseignants sur la base d'une analyse de documents d'organismes internationaux. L'ouvrage rassemble ensuite des études de cas nationales (Angleterre, Écosse, France, Roumanie, Grèce, Québec) et présente deux textes sur les standards référentiels dans l'évaluation des enseignants.

\section{ROCQUET Jean-Pol, L'inspection pédagogique aux risques de l'évaluation, L'Harmattan/Paris, 2005, 229 p., bibliogr.}

L'auteur analyse ce que recouvre le métier d'inspection et son passage d'une logique de contrôle et de vérification d'actes pédagogiques prescrits à une logique d'évaluation qui revêt selon lui autant de formes que d'évaluateurs. 


\section{L’ÉTABLISSEMENT SCOLAIRE}

\section{DUVAL Philippe, SAURAT Gérard, La fonction "conseil aux établissements}

publics locaux d'enseignement": rapport, Académie de Poitiers 2001, 70 p.

Dans ce rapport produit à la suite d'une mission d'inspection, les rapporteurs formulent différents conseils aux établissements d'enseignement. Le premier chapitre s'intéresse à la gestion depuis les règles budgétaires jusqu'au respect des règles d'hygiène et de sécurité. Le second chapitre s'attache au niveau pédagogique de l'évaluation. Les projets d'établissements sont ensuite abordés et débouchent sur l'évaluation dite "globale» des établissements tout en proposant une réflexion sur les outils de l'audit.

DUPUIS Jean-Yves, "Les contrôles sur l'établissement: dossier ", Les Cahiers de l'Éducation, septembre 2007, $n^{\circ}$ 67, p. 5-20.

Dans ce dossier consacré aux différents contrôles sur les établissements d'enseignement, tous les aspects y sont abordés depuis le chef d'établissement jusqu'à la gestion effectuée par les chambres régionales et territoriales des comptes en passant par le contrôle de la légalité des actes.

\section{EURYDICE, L'autonomie scolaire en Europe: politiques et mécanismes de mise en ouvre, Eurydice/Bruxelles, 2007, 59 p.}

L'objectif principal de cette étude est de proposer une analyse comparative des différentes formes d'autonomie scolaire mises en place aujourd'hui dans trente pays du réseau Eurydice. Elle offre une information sur les processus qui ont conduits à la dévolution des pouvoirs de décision aux écoles et sur la manière dont ces dernières sont tenues de rendre compte de leurs décisions vis-à-vis des autorités supérieures en éducation.

FORT Marc, REVERCHON-BILLOT Michel, PAIR Claude, Diriger, animer, piloter un établissement scolaire. Un état du débat, CRDP de Bourgogne, 2005, 320 .

Cette publication aborde différents thèmes concernant le pilotage de l'établissement public local d'enseignement (EPLE): sa dimension pédagogique, sa dimension juridique, mais aussi l'évolution de ce pilotage dans le cadre de la Loi organique relative aux lois de finance (LOLF), et la poursuite de la décentralisation. Enfin une ouverture sur les autres pays européens permet de mieux comprendre le fonctionnement du pilotage des établissements en France.

\section{GUSTIN André, Management des établissements scolaires: de l'évaluation} institutionnelle à la gestion stratégique, DeBoeck université/Bruxelles, 2001, 178 p., bibliogr.

Cet ouvrage propose une analyse à partir de plusieurs critères et indicateurs, en privilégiant la dimension pédagogique de l'enseignement au sein d'un établissement scolaire. Inscrivant l'évaluation institutionnelle au centre de la démarche de pilotage, l'auteur propose de dépasser le cadre objectivant de toute évaluation pour prendre en considération les facteurs psychosociaux et organisationnels. Il met en garde également contre l'insuffisance, voire l'inadéquation des contrôles de conformité qui s'appuient souvent sur des normes externes.

PELLETIER Guy, "L'évaluation des établissements scolaires: entre pilotage de régulation et pratiques d'intervention ", Revue des échanges, 2004, vol. 21, $n^{\circ} 4$, p. 29-35

Dans cet article, l'auteur porte une attention particulière à l'évolution de l'évaluation des établissements comme processus de régulation tant interne qu'externe, à l'activité éducative et montre l'intérêt que représente désormais l'évaluation comme outil de régulation. 
"Réussite des élèves, performances des établissements", Administration et éducation, 2007, $n^{\circ} 115-3,189 p$.

Ce numéro est consacré aux actes du colloque national de l'association de l'association française des administrateurs de l'éducation (AFAE) et invite à réfléchir à la notion de performance dans le domaine de l'éducation au moment où la LOLF impose de rendre compte de la performance des établissements scolaires au moyen d'indicateurs les plus pertinents possibles.

WAGNER Jean, «Évaluer pour évaluer? Évaluer pour évoluer? Évaluer pour piloter!»Revue des échanges, 2004, vol. 21, $n^{\circ}$ 4, pp. 5-9

Dans cet article, l'auteur, directeur d'un lycée technique présente ses réflexions sur les notions d'évaluation, de contrôle et de pilotage lors de la création d'un nouveau lycée technique au Luxembourg.

\section{CONTRÔLE ET ÉVALUATION DES SYSTÈMES ÉDUCATIFS - RÉGULATION DES POLITIQUES ÉDUCATIVES}

BARROSO Joao, BAJOMI Yvan, Systèmes éducatifs, modes de régulation et d'évaluation scolaires et politiques de lutte contre les inégalités en Angleterre, Belgique, France, Hongrie et au Portugal, Rapport de recherche, 2002, 73 p., bibliogr., http://www.girsef.ucl.ac.be/Deliverable3.pdf

Ce rapport s'insère dans le cadre du projet de recherche "changement des modes de régulation et production sociale des inégalités dans les systèmes d'éducation: une comparaison européenne» financé par la Commission européenne. L'objectif principal est de réaliser une recherche comparative sur l'évolution des modes de régulation publics des systèmes éducatifs secondaires dans cinq pays européens et d'analyser la façon dont ces évolutions affectent les processus locaux de production des hiérarchies et des inégalités scolaires.

BOUVIER Alain, La gouvernance des systèmes éducatifs, PUF/Paris, 2007, 360 p. (Politique d'aujourd'hui)

Dans un contexte de réformes politiques et administratives des pays, d'enquêtes et de palmarès, de logiques de résultats et de performances, les systèmes éducatifs subissent de multiples pressions. La question de la gouvernance se pose à chaque système éducatif avec ses régulations et l'utilisation d'outils du management public. À partir d'exemples concrets, l'auteur montre les sources de la gouvernance et s'interroge sur le rôle de l'État en Europe ainsi que sur les types de régulations pratiqués dans les fonctions publiques en France, en particulier dans l'Éducation nationale.

BOTTANI Norberto, MAROY Christian, MONSEUR Christian, «Évaluation des résultats scolaires: quels impacts sur les politiques d'éducation?», Politiques d'éducation et de formation. Analyses et comparaisons internationales, février 2004, $n^{\circ} 11$, p. 7-101

Les contributeurs de ce numéro jettent un regard critique sur les enquêtes internationales, leurs limites et leur évolution. Les essais rassemblés ici portent sur les composantes méthodologiques et sur l'utilisation des résultats. 
BROADFOOT Patricia, "Un nouveau mode de régulation dans un système décentralisé: l'État évaluateur ", Revue française de pédagogie, mars 2000, $n^{\circ} 130$, p. 43-55

Ce dossier est consacré au développement de l'autonomie des établissements scolaires; il analyse les mécanismes mis en place en France et en Angleterre et en particulier l'évaluation qui permet aux gouvernements de déterminer les priorités, de gérer le pilotage et de contrôler la qualité du système éducatif national.

CROS Françoise coord., BON Annette, Les évaluations en éducation au niveau international: impacts, contradictions, incertitudes. Réflexions et données extraites du séminaire international "L'évaluation au service de la qualité en éducation: pratiques et enjeux» 29 mai-2 juin 2006, CIEP/Sèvres, 2007, 172 p., bibliogr.

La première partie de cet ouvrage fait état des apports en matière d'évaluation des acquis des élèves au niveau international. La deuxième illustre le pilotage de l'école vu du niveau national et comparatif. Enfin, la dernière partie dégage les points de discussion sur tous ces dispositifs d'évaluation, aussi bien au niveau des objectifs que des normes.

«La contractualisation», Administration et Éducation, 2004, $n^{\circ}$ 104-4, $191 p$.

Après avoir défini la notion de contractualisation, les auteurs analysent les différentes formes de contractualisation à l'œuvre, entre notamment les établissements d'enseignement et les académies mais aussi avec les collectivités territoriales. Ils dégagent ensuite les enjeux liés à cet engagement et la nature de cet engagement.

DEMAILLY Lise, Évaluer les politiques éducatives: sens, enjeux, pratiques, De Boeck Université/Bruxelles, 2000, 234 p., bibliogr. (Perspectives en éducation et formation)

L'évaluation des politiques éducatives et notamment l'évaluation des établissements, des performances scolaires et du personnel, est devenue une obligation institutionnelle et morale dans la plupart des pays développés. Or, selon l'auteur, l'évaluation est un processus sociologiquement complexe qui n'engendre pas automatiquement des améliorations. À travers l'analyse d'expériences réussies ou d'échec, l'auteur propose une réflexion sur les formes les plus adaptées d'évaluation par l'action publique.

DEMAILLY Lise, VAN ZANTEN Agnès, THELOT Claude et al., "Évaluer oui mais pour quoi faire?», Nouveaux regards, mars 2002, $n^{\circ} 16$, p. 2-22

À la suite de la publication des résultats du Programme international pour le suivi des acquis des élèves (PISA) mené par l'OCDE, l'Institut de recherches de la FSU a organisé un débat avec Georges Lemaître, un responsable de ce programme et d'autres spécialistes de l'évaluation. Ce débat constitue une part importante du dossier. Il est suivi d'un article sur les nouveaux enjeux de l'évaluation et de deux entretiens, l'un d'Agnès Van Zanten, sociologue, sur le bon usage de l'évaluation et l'autre de Claude Thélot, président du Haut conseil de l'évaluation, sur les finalités de l'évaluation en éducation.

DEROUET Jean-Louis dir., NORMAND Romuald dir., L'Europe de l'éducation: entre management et politique, INRP/ESEN, 2007, 205 p., bibliogr. (Éducation Politique Sociétés)

Dans la seconde partie de cet ouvrage, les différents contributeurs analysent la mise en place d'une logique managériale en éducation dans une perspective d'intégration européenne. Un article présente les méthodes de régulation en Angleterre et aux États-Unis, un autre les points 
forts et les points faibles de l'évaluation en France en s'appuyant sur les exemples de l'Angleterre, de l'Espagne et de la Suède.

DUTERCQ Yves dir., Les régulations des politiques d'éducation, Presses universitaires de Rennes/Rennes, 2005, 204 p. bibliogr. (Des Sociétés)

Dans le contexte général de déconcentration et de décentralisation qui anime l'Europe depuis plus de vingt ans, l'auteur se penche sur les formes de régulation des politiques d'éducation au niveau supranational, national et local et cela aux différents niveaux des systèmes d'enseignement. Les contributions rassemblées apportent un regard international sur les formes de régulation et permettent de comprendre la place qu'occupe l'État désormais dans ce système.

\section{FORESTIER Christian, THELOT Claude, EMIN Jean-Claude, Que vaut l'ensei- gnement en France? Les conclusions du Haut Conseil de l'évaluation de l'école,} Stock/Paris, 2007, 293 p.

Cet ouvrage rassemble les dix-neuf avis émis par le Haut Conseil de l'évaluation de l'école créé en 2000 pour analyser l'état du système éducatif français d'une part, dresser les priorités et les évolutions souhaitables d'autre part. Après une préface de Claude Thélot, concepteur et premier président du Haut Conseil, son successeur, Christian Forestier dégage les lignes de force de cette analyse. Les conclusions du Haut Conseil sont ensuite rassemblées et commentées en chapitres. Ces chapitres abordent différents points concernant l'élève: son niveau à travers l'évaluation des connaissances, compétences et diplômes, son orientation et ses difficultés; enfin l'évaluation de l'enseignant et celle des établissements d'enseignement.

GAUTHIER Pierre-Louis coord., MICHEL Alain, COLOMBO Gianni et al., "L'évaluation des systèmes éducatifs: dossier», Revue internationale d'éducation de Sèvres, juin 2000, $n^{\circ} 26$, p. 15-129

Pratiquement inexistant dans le discours pédagogique français jusque vers 1960, le terme d'évaluation a pris depuis, une place grandissante. La demande en la matière n'a cessé de croître, à la mesure de l'effort budgétaire consenti pour une adaptation continue du système éducatif aux besoins. Ce dossier dresse un panorama de l'évaluation dans ses tendances, sa méthodologie et ses procédures. Des études de cas viennent illustrer les propos; elles concernent un certain nombre de pays comme la France, l'Italie, l'Allemagne, l'Afrique du Sud, les Pays-Bas, l'Espagne, la Grande Bretagne et la Guinée.

GAUTHIER Pierre-Louis coord., KOCH Hans Konrad, SUTHERLAND Margaret $B$ et al., "La généralisation de l'évaluation contribue-t-elle à améliorer la qualité de l'enseignement», Revue internationale d'éducation de Sèvres, décembre 2005, $n^{\circ} 40$, p. 25-41

Le numéro, intitulé «l'éducation dans le monde: débats et perspectives», rend compte des études de cas et des analyses présentées par des experts français et étrangers qui ont participé aux «Rencontres internationales» organisées à Sèvres du sept au neuf avril 2005 sur le thème «mieux comprendre l'école de demain, dix années d'éducation dans le monde». L'un des ateliers propose une réflexion sur l'évaluation à travers cinq études de cas: Allemagne, Grande-Bretagne, Sénégal, France et Catalogne.

LANDSHEERE Gilbert de, Le pilotage des systèmes d'éducation, De Boeck Université/Bruxelles, 1994, 192 p., bibliogr. (Pédagogies en développement)

L'auteur livre une réflexion générale sur la nature du pilotage et ses orientations majeures puis précise ce que sont les indicateurs et comment les utiliser. Les principales réalisations nationales et internationales en matière de macropilotage et micropilotage sont décrites en précisant leurs avantages et leurs inconvénients. Parmi les pays étudiés se trouvent les États-Unis, l'Angleterre, la France, le Canada, la Suède, l'Australie, le Danemark et la Nouvelle-Zélande. 


\section{MAROY Christian, École, régulation et marché. Une comparaison de six espaces locaux en Europe, PUF/Paris, 2006, 336 p.}

Sous différentes influences, qu'elles soient internationales, économiques ou sociales, les gouvernements européens cherchent à changer les modes de régulation de leurs systèmes éducatifs pour plus d'efficacité et d'équité. Certains estiment que le marché et le libre choix des parents peuvent améliorer le fonctionnement des écoles alors que d'autres parient sur l'évaluation et la régulation par les résultats. Partout, de nouveaux outils de régulation apparaissent, poussés par de nouveaux acteurs. Cet ouvrage, issu d'une recherche européenne menée dans plusieurs pays européens, établit un état des lieux des tendances en cours et analyse leur genèse et leurs effets au plan local. Il s'appuie sur la comparaison des politiques nationales et sur l'analyse de six espaces scolaires: Budapest, Charleroi, Lille, Lisbonne, Londres et la région parisienne.

\section{MEURET Denis, Gouverner l'école - une comparaison France/États-Unis, PUF, 2007, 232 p. (Éducation et société)}

L'auteur propose une réflexion sur le gouvernement des systèmes scolaires. Sous ce concept, il englobe à la fois la gestion du système éducatif dans la capacité à atteindre les objectifs fixés au préalable mais aussi la conception de l'éducation formelle. Sa réflexion s'appuie sur une comparaison entre la France et les États-Unis. La première partie de cet ouvrage porte sur les objectifs des deux écoles et sur le rôle et le contenu de l'éducation; la seconde partie approfondit la problématique de la régulation de l'éducation à travers différents thèmes comme l'autonomie des établissements scolaires, le pilotage par les résultats et les logiques d'inspection.

MONS Nathalie, PONS Xavier, Les standards en éducation dans le monde francophone: une analyse comparative, IRDP/Neuchâtel/Suisse, 2006, 149 p., bibliogr.

Les politiques d'évaluation standardisées obéissent à des philosophies et à des logiques opératoires diversifiées. Après une tentative de définition du terme "standard», concept anglosaxon, les auteurs décrivent les politiques mises en œuvre dans quatre pays francophones: la France, la Belgique, la Suisse et le Québec. Le troisième chapitre présente des facteurs explicatifs du développement de cette culture de l'évaluation en éducation. Enfin, les auteurs se penchent sur les effets de ces politiques, constatant la rareté des recherches qui y sont consacrées, ainsi que sur leurs enjeux.

OCDE: Organisation de coopération et de développement économiques/Paris, Regards sur l'éducation 2007: les indicateurs de l'OCDE, OCDE/Paris, 2007, 471 p., annexes

Ce rapport annuel, organisé thématiquement, présente un ensemble d'indicateurs actualisés, quantitatifs et comparables sur les résultats des systèmes d'enseignement des pays de l'OCDE. Ces indicateurs rendent compte des résultats des établissements d'enseignement et de l'impact de l'apprentissage; des ressources financières et humaines investies dans l'éducation; de l'accès à l'éducation, de la participation et la progression des élèves et étudiants; enfin, de l'environnement pédagogique et de l'organisation scolaire. L'édition 2007 s'intéresse plus particulièrement à la qualité des résultats de l'éducation, aux leviers politiques et aux circonstances qui conditionnent ces résultats, ainsi qu'aux différentes formes de rendement privé et social généré par les investissements en éducation.

\section{PELLETIER Guy, Autonomie et décentralisation en éducation: entre projet et évaluation, Éd. de l'AFIDES/Montréal, 2001, 159 p.}

À travers plusieurs contributions, cet ouvrage présente les grands axes de réflexion actuels concernant l'organisation du système éducatif, son pilotage et sa régulation. Il analyse les 
nouveaux enjeux de pouvoir et les changements qui s'opèrent notamment au niveau du personnel d'encadrement des ministères, des régions et des établissements.

"Piloter par les résultats», Administration et éducation, 2003, n 98-2, 179 p. Les différentes contributions de ce numéro proposent une réflexion sur la notion de pilotage, son émergence et son développement. Sont également abordées les notions de régulation et de gouvernance du système éducatif.

"Regards sur la LOLF», Administration et éducation, 2007, $n^{\circ} 113-1,179 p$. Ce numéro consacré à la LOLF se place dans une perspective historique et comparative; les différents auteurs évoquent ainsi sa mise en place et montrent comment sa réussite nécessite une amélioration fondamentale des modes de pilotage entre les différents niveaux du système éducatif.

ROBINSON Muriel, "Le pilotage par objectifs en Angleterre: pratiques et problématiques", Revue internationale d'éducation Sèvres, décembre 2007, $n^{\circ} 46$, p. $132-140$

Dans cet article l'auteur présente la mise en place d'une politique de gestion des performances au sein d'un établissement et agréé par les directives gouvernementales. Elle définit dans un premier temps les grandes lignes du nouveau système tel qu'il va être mis en place et tente de répondre aux questions concernant les liens entre pilotage par objectifs et priorités pédagogiques, la mise en place de la régulation entre établissements et les transformations que ce pilotage induit.

\section{SITES INTERNET}

\section{Standing International Conference of national and regional Inspectorates of Éducation (SICI)}

Cette organisation, créée en 1995, regroupe les inspections nationales et régionales des différents pays d'Europe, compétentes en matière d'éducation. Elle compte actuellement vingtcinq membres. Elle contribue au développement de l'éducation en Europe en produisant des données et des analyses fondées sur l'observation directe des pratiques dans les écoles. Les inspections jouent des rôles très différents selon les pays, elles ont toutefois le mandat d'évaluer la qualité des écoles et de l'éducation en général. Elles ont en commun un statut public ainsi qu'un financement étatique ou régional dans les systèmes décentralisé. http://www.sici-inspectorates.org/

\section{Eurydice}

Outre la base de données proposée en ligne qui permet des recherches par mots-clés sur le contrôle et l'évaluation dans les différents pays et sur les différents niveaux du système éducatif, le site présente de nombreuses informations sur l'éducation en Europe. Il présente les dernières publications d'Eurydice. http://www.eurydice.org/ 
